\title{
The Origins of Planetary Nebulae in the Inner and Outer Disks of M 31
}

\author{
Bruce Balick $^{1}$, Karen Kwitter ${ }^{2}$, Romano Corradi ${ }^{3,4}$, \\ Rebeca Galera Rosillo ${ }^{4,5}$ and Richard Henry ${ }^{6}$
}

\author{
${ }^{1}$ Astronomy Department, University of Washington, Seattle WA, 98195-1580, USA \\ ${ }^{2}$ Department of Astronomy, Williams College, Williamstown MA, 01267-2565, USA \\ ${ }^{3}$ Gran Telescopio de Canarias, Center for Astrophysics in La Palma, S/C de Tenerife, E38712, \\ Spain \\ ${ }^{4}$ Departamento de Astrofisica, Universidad de La Laguna, La Laguna Tenerife, E-38206, Spain \\ ${ }^{5}$ Instituto de Astrofisica de Canarias, La Laguna, Tenerife, E-38205, Spain \\ ${ }^{6}$ Richard Henry, H. L. Dodge Department of Physics \& Astronomy, University of Oklahoma, \\ Norman, OK 73019, USA
}

\begin{abstract}
The planetary nebulae (PNe) of M 31 are receiving considerable attention as probes of its structure and chemical evolution in a galactic environment that is putatively similar to the Milky Way. We have obtained deep spectra for about 30 luminous PNe in M 31's inner disk and beyond $\left(\mathrm{R}_{g a l}<105 \mathrm{kpc}\right)$. The entire ensemble of $\mathrm{PNe}$ exhibit $\mathrm{O} / \mathrm{H} \sim 2 / 3$ solar with no discernible radial gradient, in stark contrast to the H II regions of M 31. This suggests that the outer PNe in M 31 formed from a common O-rich ISM at least 5 GY ago. We infer that the outer PNe and the underlying stellar population have little common history in $\mathrm{M} 31$, and that the formation of the O-rich PNe preceded any putative encounter with M $33 \sim 2-3$ Gy ago.
\end{abstract}

Keywords. planetary nebulae: general, galaxies: abundances, galaxies: halos, ISM: evolution

\section{Introduction}

Undisturbed spiral galaxies exhibit a standard form consisting of stellar ensembles with distinct morphologies (bulges, disks, and halos). Presumably each of these ensembles shares distinct histories across the class of spirals. Their differences in shapes are echoed by their distinct kinematic patterns: stars in disks rotate as a whole about the symmetry axis of the disk whereas halo stars follow elongated orbits with random orientations. The stars of the nearest regular spiral galaxy, M31, also follow this pattern, though some faint streams of stars extend in projection out to about $100 \mathrm{kpc}$ ( e.g., Ibata et al. 2001, Majewski et al. 2003, Ibata et al. 2014).

Thus we imagine that M31 is a fairly normal spiral galaxy with a typical formation history. Its proximity facilitates observational studies of M 31's present stellar distribution and kinematics. Kinematic studies, coupled with large-scale abundance studies, allow us to glimpse the evolutionary history of M 31 in detail. Like nearly all spirals, the substantial differences in metallicity of stars in the disk and the halo of M 31 support the idea that the formation and evolutionary histories of both stellar ensembles are distinct and fairly characteristic outcomes of the evolutions of all spirals. More to the point here, large-scale studies of M 31 are especially valuable since the origins of thick disks and halos of spirals are still somewhat unclear (cf. Yoachim \& Dalcanton 2005, Bernard et al. 2015).

Gaseous tracers are often more easily observable probes of galactic structure kinematics and abundances than are stars, especially in the inner disk of M31 where H II regions are numerous. Most planetary nebulae ("PNe") and virtually all $\mathrm{H}$ II regions lie inside its 
photometric radius $R_{25}=20.6 \mathrm{kpc}$. The H II regions in M 31 display roughly solar metal abundances with mild radial gradients (Zurita \& Bresolin 2012), much like other spirals of comparable size and mass (cf. Magrini et al. 2016 and various references within).

$\mathrm{PNe}$ are the only gaseous tracers of alpha-element abundances (such as $\mathrm{O}$ and $\mathrm{Ne}$ ) beyond $R_{25}$ in M31. Their luminous emission lines $\left(\sim 10^{4} \mathrm{~L}_{\odot}\right)$ render them easily identifiable, useful for tracing bulk kinematics, and viable for measuring metal abundances and their large-scale gradients in spirals. In this paper we use PNe throughout M31, including the region of the halo - and wherever stars younger than $\sim 10^{10} \mathrm{y}$ are found-to trace the abundance of $\mathrm{O} / \mathrm{H}$ and surmise its chemical history.

An extensive PN identification survey of PNe in M 31 using [O III] fluxes was published by Merrett et al. (2006, "M+06"). In their analysis they found over $2700 \mathrm{PNe}$ in M 31 whose kinematics are distinct from the stellar streams, M32, and dwarf galaxies in their fields. As expected, the PNe in this sample follow the distribution of red giants. Also the luminosity functions of the PNe follow a universal pattern at all radii where statistics can be compiled. Somewhat oddly, however, $\mathrm{M}+06$ showed that most of the PNe beyond $\mathrm{R}_{25}$ display an ensemble pattern of kinematics similar to that of the PNe in inner disk and the HI rotation curve. A few PNe are found in this "exodisk" at even larger radii. Almost none of the entire sample have the kinematics expected of a halo population.

\section{Observations \& Methodology}

PNe selected from the $\mathrm{M}+06$ catalogue of $\mathrm{PNe}$ in $\mathrm{M} 31$ have a complete set of observable optical nebular oxygen line fluxes that have long been used for determining $\mathrm{O} / \mathrm{H}$ in the PNe of the Milky Way and the Magellanic Clouds. Accurate O/H abundances require good flux measurements of the faint [O III] $] 4363$ line. To this end we used 8-10-m class telescopes for PNe lying within about two magnitudes of the most luminous [O III] emitters (aka " $\mathrm{M}_{*} \mathrm{PNe")}$. The $\mathrm{O} / \mathrm{H}$ abundances of all targets have been consistently derived using well-understood methodology (e.g., Kwitter \& Henry 2001, Henry et al. 2004). Ionization models argue that selecting very [O III]-luminous PNe does not induce a strong bias in the outcome of $\mathrm{O} / \mathrm{H}$ values.

Most of the data in this study were obtained using the 8.4-m Gemini-North Telescope (see Kwitter et al. 2012 for details) and the 10.4-m Gran Telescopio Canarias (see Balick et al. 2013 and Corradi et al. 2015). Nearly all of the targets are kinematic members of M31's exodisk. However, last year we obtained spectra of an additional six PN spectra of luminosity-selected PNe in M 31 that are mostly within $R_{25}$. These are included here (Galera-Rosillo, 2016, this volume).

Values of $\mathrm{O} / \mathrm{H}$ derived from our measurements are shown in Fig. 1. The most recent results are shown as large orange dots and earlier measurements as red and blue dots. Blue dots identify $\mathrm{PNe}$ that do not have disk- or exodisk-like kinematics. The solar abundance is indicated as a horizontal line. A linear gradient fitted to the red and orange dots is not robust: that is, the sign of its slope varies when any third of the data points are removed. (Even the sign of the slope may well change with new data.)

One straightforward conclusion is that the ensembles of disk and exodisk PNe in our study share an unexpectedly large $\mathrm{O} / \mathrm{H}$ abundance and an insignificant radial gradient throughout $\mathrm{M} 31$. Indeed, $<[\mathrm{O} / \mathrm{H}]>\sim-0.2$, far in excess of the metallicity measured in the underlying stellar population beyond $\mathrm{R}_{25},<[\mathrm{Fe} / \mathrm{H}]>\sim-0.7$. Given this and the fact that the kinematics of genuine halo stars and PNe in the exodisk show very different aggregate kinematic properties, we conclude that the histories of these stellar populations have little in common. This may also apply within the disk of M 31 also (Magrini et al. . 


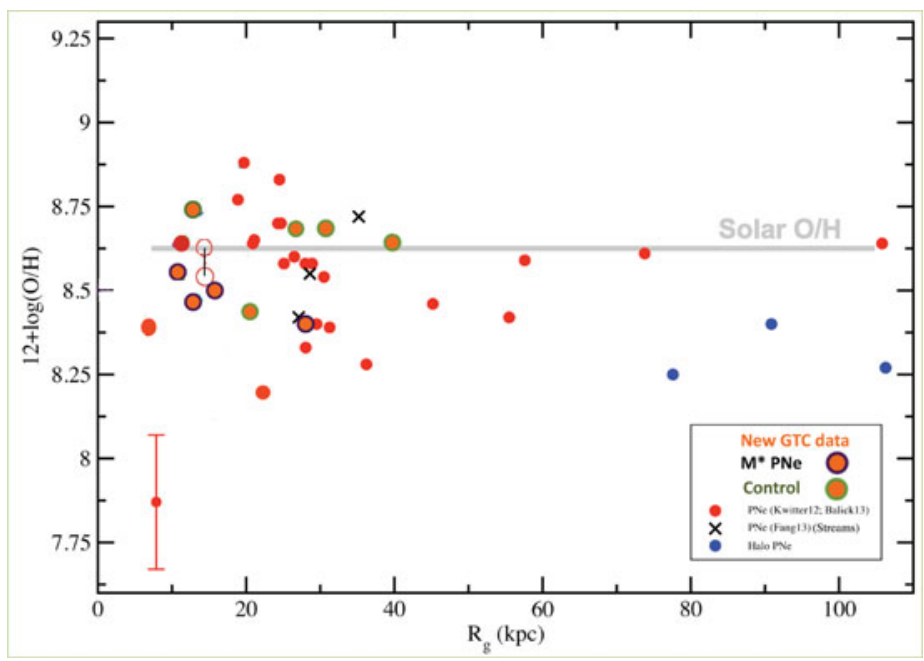

Figure 1. Our $\mathrm{O} / \mathrm{H}$ measurements of $\mathrm{PNe}$ in $\mathrm{M} 31$ as a function of deprojected disk radius (dots). See text for details.

The exodisk PNe are otherwise chemically ordinary. Their $\mathrm{Ne} / \mathrm{O}$ and $\mathrm{He} / \mathrm{H}$ ratios are typical of those in the Milky Way and the Magellanic clouds. One the other hand, initial masses of the progenitor stars lie well below the $\sim 4-\mathrm{M}_{\odot}$ ) limit for enriching nitrogen or helium (Karakas \& Lattanzio). Hence the highly unusual values of $\mathrm{O} / \mathrm{H}$ and $\mathrm{Ne} / \mathrm{H}$ must have originated in the ISM from which the progenitor stars of the PNe formed.

We note that two PNe in the sample appear to have a high $\mathrm{N} / \mathrm{O}$ abundances $(\geqslant 0.7)$. However, their [NII]6584/6548 flux ratios depart substantially from 2.9 so the anomalous $\mathrm{N} / \mathrm{O}$ ratios need independent confirmation.

Finally, our result of near-solar O/H for the PNe of M31's exodisk are probably not replicated in other large spiral galaxies (Magrini et al. . Accordingly, the historical origin of these PNe in M31 is likely to be different from those in other undisturbed spiral galaxies. The kinematics of PNe beyond $R_{25}$ in other spirals is a good test this assertion.

\section{Interpretation \& Conclusions}

To summarize, our abundance results coupled with aggregate kinematic data suggest that (with only minor exceptions) the entire ensemble of PNe presently located at $5<R_{\text {gal }}<100 \mathrm{kpc}$ formed from a large-scale ISM characterized by nearly solar alphaelement abundances. No trace of this ISM remains; it exists today only in the inner 10 kpc of M31's inner disk (e.g., Zurita \& Bresolin, but see also Olsen et al. 2006).

Hence the PN formation process may not have been steady throughout M 31's lifetime but rather occurred during some sort of an unusual global enrichment event. On the other hand, as pointed out to us privately by Harriet Dinerstein, there are other nuclear pathways for enriching oxygen. (However, such mechanisms don't apply to the PNe in other spiral galaxies, including in the Milky Way where $\mathrm{PNe}$ exhibit values of $\mathrm{O} / \mathrm{H}$ that are similar to H II regions and red giant stars.)

We have used our ionic abundances to constrain CLOUDY models that make it possible to estimate the luminosities and temperatures of the central stars of the $\mathrm{PNe}, \mathrm{L}_{*}$ and $\mathrm{T}_{*}$, respectively. Some of this work is still underway. We have plotted the preliminary results on an $\mathrm{H}-\mathrm{R}$ diagram with evolutionary tracks that indicate the final masses and ages of the central stars. When comparing our points to new nearby tracks of the post-AGB 
evolution of central stars by Miller Bertolami (2016) we find characteristic initial stellar masses lie in the range $\sim 1.2-1.3 \mathrm{M}_{\odot}$. Hence the PNe in our study have ages $\geqslant 5$ Gy. So the ISM from which the central stars formed was probably in place well before a putative encounter between M 33 and M 31 2-3 Gy ago (McConnachie et al. 2009) that initiated the stellar streams threading the halo of M 31 (cf. Ibata et al. 2014).

The same exodisk kinematic pattern applies an extended stellar disk of red giant stars at $R_{\text {gal }}>40 \mathrm{kpc}$ by Ibata et al. 2005, Chapman et al. 2006. Thus the population PNe in our study has a stellar counterpart, most of which exhibit $[\mathrm{Fe} / \mathrm{H}]$ values closer to that expected of old halo stars: $\leqslant-0.7$ (Chapman et al. Richardson et al. Hammer et al. 2010).

In the preceding talk Dr. Xuan Fang reviewed O/H abundances of the M31's PNe located in its outer stellar streams. Dr. Fang independently finds very similar O/H abundances and gradients as we find in M 31's exodisk. We surmise that although the PNe that he studied may have formed from the same metal-rich ISM as did ours, they could have acquired the kinematics of the streams during a subsequent encounter with M 33-M 31.

While the putative M31-M 33 encounter is a convenient way to account for the kinematics of the ensemble of PN beyond $R_{25}$, it may not actually be relevant. Note that all but three of the exodisk PNe have relaxed into disk-like orbits. The relaxation time of a star at an orbital radius of $100 \mathrm{kpc}$ is approximately its orbital time, $2 \mathrm{GY}$. That is, the kinematics of PNe were probably established prior to an encounter (and any remnants of the metal-rich ISM from which they formed may well have been stripped and ejected).

\section{References}

Balick, B., Kwitter, K. B., Corradi, R. L. M., \& Henry, R. B. C. 2013, ApJ, 774, 3

Bernard, E. J., Ferguson, A. M. N., Richardson, J. C., et al. 2015, MNRAS, 446, 2789

Chapman, S. C., Ibata, R., Lewis, G. F., et al. 2006, ApJ, 653, 255

Corradi, R. L. M., Kwitter, K. B., Balick, B., Henry, R. B. C., \& Hensley, K. 2015, ApJ 807, 181

Hammer, F., Yang, Y. B., Wang, J. L., et al. 2010, ApJ, 725, 542

Henry, R. B. C., Kwitter, K. B., \& Balick, B. 2004, AJ, 127, 2284

Ibata, R., Chapman, S., Ferguson, A. M. N., et al. 2005, ApJ, 634, 287

Ibata, R., Irwin, M., Lewis, G. F., \& Stolte, A. 2001, ApJ (Letters), 547, L133

Ibata, R. A., Lewis, G. F., McConnachie, A. W., et al. 2014, ApJ, 780, 128

Kwitter, K. B. \& Henry, R. B. C. 2001, ApJ 562, 804

Karakas, A. I. \& Lattanzio, J. C. 2014, PASA, 31, e030

Kwitter, K. B., Lehman, E. M. M., Balick, B., \& Henry, R. B. C. 2012, ApJ, 753, 12

Majewski, S. R., Skrutskie, M. F., Weinberg, M. D., \& Ostheimer, J. C. 2003, ApJ, 599, 1082

Magrini, L., Coccato, L., Stanghellini, L., Casasola, V., \& Galli, D. 2016, A\& A, 588, A91

McConnachie, A. W., Irwin, M. J., Ibata, R. A., et al. 2009, Nature 461, 66

Merrett, H. R., Merrifield, M. R., Douglas, N. G., et al. 2006, MNRAS, 369, 120

Miller Bertolami, M. M. 2016, A\&A, 588, A25

Olsen, K. A. G., Blum, R. D., Stephens, A. W., et al. 2006, AJ, 132, 271

Richardson, J. C., Ferguson, A. M. N., Mackey, A. D., et al. 2009, MNRAS, 396, 1842

Vassiliadis, E. \& Wood, P. R. 1994, ApJS, 92, 125

Yoachim, P. \& Dalcanton, J. J. 2005, ApJ, 624, 701

Zurita, A. \& Bresolin, F. 2012, MNRAS, 427, 1463

\section{Discussion}

OTsukA: I think that the estimation of the current luminosity and core-mass of the central star and the initial mass based on the post-AGB evolution tracks depends on 
the shape of the adopting SED of the central star. Did you adopt the theoretical stellar atmosphere model? or blackbody SED?

BALICK: We adopt SED models from the options available within CLOUDY. Since I don't run the models I will guess that we adopt blackbody models for the emergent UV spectrum and then vary the stellar luminosity until the CLOUDY-estimated line fluxes and ratios agree with our observations.

Q: Could the halo have formed from the interaction with the dwarf galaxy which Kniazev et al. (2014) claim left a remnant in the halo, at M31 NE?

BALICK: Possible, but unlikely. Small (dwarf) galaxies have small metal abundances $([\mathrm{O} / \mathrm{H}] \sim<0.3)$. What's more, I would guess that the numbers of assimilated dwarfs would be unreasonably large given the numbers of known dwarfs today. In addition, these encounters might not explain the disk-likekinematics of the ensemble of $\mathrm{PNe}$ at large radii from M31's nucleus. Finally, it would be nice if an evolutionary scenario accounted for the presence of an $[\mathrm{O} / \mathrm{H}]$ gradient that is flat and continuous between projected nuclear distances from the inner disk $(\sim 10 \mathrm{kpc})$ to the survey limit $(\sim 100 \mathrm{kpc})$.

STERling: You mentioned that the PNe observed appear to be too old to be from the starburst 3 Gyr ago. Is this based on the estimated central star masses? If so, how did you estimate the luminosities and temperatures of the central stars for mass determinations?

BALICK: Yes, the estimate is based on estimates of $\mathrm{L}^{*}$ and $\mathrm{T}^{*}$ based on (1) our spectra; (2) emission-line luminosities; and (3) most of all CLOUDY models that successfully predict the observables using mass and temperature models of central star ionizing radiation. 